\title{
Session des 19 et 20 novembre 1959
}

Le Comité Technique de la Société Hydrotechnique de France s'est réuni à Paris les 19 et 20 novembre 1959 , au cours de trois séances:

- 2 séances spéciales, consacrées au thème : «La Mécanique des fluides et la Météorologie $»$, organisées avec le concours de la Société Météorologique de France, le 19 novembre, 12, place des Etats-Unis $\left(16^{\circ}\right)$;

- 1 séance réservéc aux comptes rendus de récents congrès d'hydraulique, te 20 novembre, 7 , rue Lapérouse $\left(16^{\mathrm{e}}\right)$

Ont participé à cette session :

a) Conseil d'administration de la S.H.F.

M. Barnillon, Président d'honnetr";

M. Delattre, Président:

M. Thimen, Membre du Bưreau;

MM. de Rouvrles, Gridel et Remenieras;

II. le Directeur du Génie Rural et de l'Hydraulique Agricole, représenté par M. Carlerer;

M. Corne, représenté par M. Legnos.

b) Comité technique de la S.H.F. :

ar. Remenifras, Secrétaire général du Comité Technique; Min. Arlery, Aunoy, Banal, Baticle, Beau, de BeaureGatrd, Berkaloff, Bindel, Bonnefille, Bonner, Boyche't, Bourguigon, Calllez, Cappus, Chabert, Cha mayou, Charmonnier, Deymí, Drovhis, Duguennois, Dénanton, FaUconNer, FaUte, FErry, Frolow, Gaspard, Gibert, Govgenhem, Houpeunt, Hug, Kammerlocher, Kampe de Feriet, KaAvTChenko, Larietoutle, Laflèche, Lamouroux, Langlois, Larrieu, Lifeber, Lombardi, Lugiez, Melot, Mesnager, Perret, Reneuve, Roulleau, Roycourt, Serra, Terrassa, Mario Tonini, Valembois, de Saint-Vaulry;

M. Lyon, représenté par M. LABYE;

M. HeNin, représenté par M. 'Iunc.

c) Adhérents :

Electricité de France représentée par: MM. Argand, Burel, Chevalier, Duboe, Duczos, Dumaine, Guilhot, Guillemin, Guillot, JacQUet, Linkovskr, Marinier, Mhlowowski, ONonko, Pauline, Preyka, Play, Roger, Rogrssard, TaBET;

Le Commissariat à l'Energie Atomique, représenté par M. LE QUnNo;
Les Ateliers de Vevey représentés par M. Graeser;

La Cie Industrielle de Travaux et d'Etudes, représentée par M. Patin;

Les Travaux Souterrains, représentés par M. Uguet;

L'Institut de Mécanique des Fluides de Toulouse, représenté par M. Thirnotor.

d) Invités par la S.H.F.:

MM. Drephuis et Kolkman du Laboratoire d'Hydraulique de Delft;

Le Laboratoire Central d'Hydraulique de France, représenté par MM. Mrgniot et Molguilny;

Institut Français du Pétrole, représenté par M. IfrLy

ir. Droumin de la Cie Saint-Gobain;

MM. Benthier, Chabert d'Hik̀res, Cocombles, Oudiv, Rinex;

M. Revol, de La Houille Blanche.

e) Membres de la Société Météorologique de France et personnalités invitées par cette Société.

Mmes Roulleav et Varllant, du C.N.R.S.;

Mme NeTTER;

Mle Nonn, de l'Observatoire du Parc Montsouris;

MM. Dabry, Forestien, Gatineau, de la Cie Air-France

N. Bricard, Professeur à la Faculté des Sciences;

M. Quenez, Professeur à la Sorbonne;

M. Duvergé, du Centre Météorologique d'Orly;

M. Durand Onstes, Assistant à l'Institut de Géographic

M. Dousser, Ingénieur à «Sud-Aviation»;

M. GeNTY, Colonel (C.A.S.D.N.);

M. Coneau, Officier d'Artillerie;

M. SAINT-Guilx, du Museum d'Histoire naturelle;

M. Tschirhard, du S.R.E.A.;

Miv. Baratoux, Beaufils, Beau, Búmenger, Bument, Bhichambault, Carton, Dady, Fontaine, Genève, Giao, Grraud, Jeunet, Lacaze, Lambert, Le bras, Marteau, Mezin, Pone, Rouadd, Saissac, Schlemberger, Tasson, Théron, Thomas, Joannès Thomas, de la Météorologic Nationale.

\section{S'étaient excusés :}

M. Gibrat, Président du Comité Technique;

Mi. Viaut, Bernard Renaud, Bafour, Balseinte, Carlleux, Crausse, Davidenkoff, Emsalem, Haegelen, Lescalt, Ligouzat, Merier, Messines du Sourbier, Morlat, Péguy, Ract-Madoux, Rodier, Schlag, Schijf, Thiusse, Wirengo, Vincent (Georges).

\section{SÉANCE DU JEUDI MATIN 19 NOVEMBRE 1959}

La séance est ouverte a 9 heures sous la présidence de M. Chapouthen, Inspecteur général à Electricité do France.

M. le President fait part des excuses des membres qui nous ont exprime leurs regrets de ne pouvoir assister cette session. En premier lieu, il présente les excuses de M. R. Grarat, Président du Comité Technique, qui, pour la première fois depuis cinq années de présidence, se trouve empêché par l'obligation impérieuse d'un voyage inopiné.

Puis M. le Président se fait un agréable devoir de saluer un certain nombre de collègues étrangers qui, comme i chacune de nos sessions, honorent nos réunions de leur présence : ce sont les représentants de M. THIJsse, MM. Diephuis et Kolkman, du Laboratoire d'Hydraulique 
de Delft (Hollande) et M. Mario Tonine, de la Societa Adriatica di Elettricita (Venise), et peut-etre d'autres encore que M. le Président s'excuse de ne pas citer, ne les ayant pas encore repérés dans l'assistance.

\section{VIE DE LA SOCIÉTÉ}

\section{Approbation du procès-verbal de la session des 18 et 19 Juin 1959}

Le procès-verbal de la session des 18 et 19 juin 1959 , déposé au bureau de la présente session, est approuvé, aucune observation n'ayant été présentée au cours de la séance.

Ce procès-verbal est publié dans le présent numéro (voir sommaire).

\section{Nécrologie}

M. le Président fait part du décès de plusieurs membres de la:S.H.F. :

- M. Thaller, Inspecteur général honoraire d'Electricité de France, Membre du Comité Technique et des Sections «Génie civil » et «tude des débits de crues», décédé le 30 décembre 1958 ;

- M. Berfils, Ingénieur à la Société Alsthom, Membre de la Commission d'études pour la répartition des PD², décédé le 6 février 1959;

- M. SuqueT, Inspecteur général des Ponts et Chaussées en retraite, Membre du Comité Technique et des Sections «Génie civil » et «Etude des débits de crues», décédé le 6 juillet 1959 ;

- M. Parmentren, ancien Vice-Président du Conseil général des Ponts et Chaussées, Membre du Comité Technique ef de plusieurs Sections (Hydraulique générale, Génie civil, Etude des débits de crues), décédé le 6 septembre 1959 ;

- M. Cavener, Inspecteur général des Ponts et Chaussées, Membre du Comité Technique et de la Section d'Hydraulique Fluviale et Maritime, décédé le 16 octobre 1959 ;

- M. Dodero, Sous-Directeur de l'Ecole Nationale Supérieure d'Electrochimie de Grenoble, auteur d'une méthode de titrage des solutions au bichromate pour la mesure des débits par dilution, présentée au Comité Technique, en collaboration avec MM. SILnen et Dumas, le 19 mars 1948. M. Dodeno est décédé subitement le 25 septembre 1959 au cours d'une seance du Congrès des Electriciens d'Aix-les-Bains.

En même temps que nos condoléances aux familles de nos membres regrettés, nous présentons nos excuses pour les omissions dont nous pourrions nous rendre coupables dans cette nécrologie, qui n'est portée à notre connaissance que d'une manière très fortuite.

\section{Distinctions honorifiques}

M. le Président fait part des distinctions qui honorent un certain nombre de nos collègues :

- M. Pierre AllleneT, Directeur général adjoint d'Electricité de France, Vice-Président de la S.H.F., a été promu au grade de Commandant de la Légion d'honneur.

Ont été promus au grade d'Officier de la Légion d'honneur :

- M. Marcel Mary, Contrôleur général à E.D.F., Membre du Conseil d'Administration et du Comité Technique de la S.H.F.;
M. Didier Oblvikl-Makra, Directeur de l'Equipement a E.D.F., Membre du Conseil d'Administration et du Comite 'Technique de la S.H,F.;

-- M. Louis Saurceor, Directeur de l'Electricité et du Gaz au Ministère de l'Industrie et du Commerce, Membre de notre Conseil d'Administration et de notre Comité Technique;

- M. Jean Goguel, Directeur des Services de la Carte géologique de la France, Rapporteur général de la Question V (Eaux minérales) aux VIes Journées de l'Hydraulique;

- M. Marcel Roubault, Directeur de l'Ecole Nationale Supéricure de Géologie de l'Université de Nancy, Rapporteur général de la Question IV (Eaux souterraines dans les mines) aux VI ${ }^{\circ}$ Journées de l'Hydraulique.

Enfin, M. René Colas, Directeur de l'Association Française pour l'Etude des Eaux, Membre du Comité Technique, a été nommé Chevalier de la Légion d'honneur.

D'autre part, M. Jean Messines du Sourbier, Inspecteur général des Eaux et Foréts à Grenoble, Président de notre Section «Glaciologie », s'est vu décerner par l'Académie des Sciences le Grand Prix de Géographie, pour ses travaux de reconstitution des forêts et de défense des sols en Chine.

Le Bureau du Comité Technique adresse à tous ses bien vives félicitations.

\section{Admission au Comité Technique}

Le Bureau propose d'admettre comme membres du Comité Technique :

- M. Vazeille,

- M. Cazenave,

- M. Mauchanir,

tous trois Ingénicurs à la Compagnie Nationale du Rhône;

-- M. Texien, Directeur du Service Technique de l'Energie Electrique et des Grands Barrages au Ministère de l'Industrie et du Commerce;

-. M. Jacever, Ingénieur à la Division Hydrologie du Service des Etudes et Recherches hydrauliques d'Electricite de France;

- M. Duport, Chef du Service des Etudes des Machines a la Société Grenobloise d'Etudes et d'Applications Hydrauliques (S.O.G.R.E.A.H.).

\section{ACTIVITÉ}

M. le Président rend compte de lactivité des diverses sections de la Société, de ses publications et des informations qu'elle a reçues depuis la dernière session.

\section{Section d'Hydraulique Générale}

La Section d'Hydraulique générale s'est réunie le 18 novembre 1959 sous la présidesce de M. Escande, poul entendre et discuter les communications de :

a) M. Ponsar, Ingénieur à la Société Hydraulique el Urbanisme sur : «Un systeme de calculs continus par analogie hydrostatique $\gg$;

b) Mme Guyot, MM. Novgaro et Thirriot, du Laboratoire d'Hydraulique de l'Ecole Nationale Supérieure d'Electrotechnique, d'Electronique et d'Hydraulique de Toulouse sur : «Contribution à l'étude numérique des régimes transitoires dans les canaux $\gg$ (utilisation des machines électroniques pour résoudre ces problèmes par différences finies). 


\section{Tournée Glaciologique \\ et de Documentation Hydroélectrique 1959}

La Tournée Glaciologique et de Documentation Hydroélectrique s'est déroulée avec un plein succès, du 20 au 24 juillet 1959 , dans les massifs des Grandes-Rousses et de Peclet-Polset, suivant le programme qui a été donné a notre dernière réunion.

Le triple intérêt documentaire de ce voyage a eté atteint :

a) Hydroélectricité, par la visite des travaux d'aménagement du Drac (barrage de Monteynard), l'exposé de M. Combe et M. Levrat sur le projet d'aménagement de la Romanche, et la visite du site du futur barrage de Grand-Maison, dans la vallée de l'Eau et d'olle, qui constituera la pièce maîtresse de cet aménagement;

b) Glaciologie, par la visite des glaciers de Sarennes et de Gébroulaz;

c) Nivologie, par la visite des travaux de protection contre les avalanches et de reboisement dans les vallées des Villards et du Bugeon et à la série domaniale de Celliers.

\section{Bureau du Comité Technique}

Le Bureau s'est réuni les 18 juin, 17 septembre et 15 octobre pour préparer la présente session et les activites prochaines de la S.H.F., qui sont les suivantes :

\section{Session de Mars 1960}

Cette session sera consacrée au thème général : « Ia mécanique des émulsions (liquide-gaz et liquide-liquide) $\gg$; nous comptons dès maintenant sur la contribution d'ume société de transport pétrolier et d'Electricité de France, et nous espérons une participation de personnalités du Génie chimique et du Commissariat à l'Energie atomique.

Nous prions les membres du Comité Technique qui aturaient des mémoires à présenter sur cette question de nous adresser dans un bref délai un sommaire d'une dizaine de lignes pour que le Bureau puisse établir, en temps voulu, un ordre du jour cohérent.

\section{VI ${ }^{\text {ss }}$ Journées de l'Hydraulique : "L'Hydraulique Souterraine "}

Près de 90 rapports nous ont été proposés. Les rapporteurs généraux se réuniront le 24 novembre pour en nccepter un certain nombre et les classer dans les diflérentes questions groupées sous le thème général : « L'Hydraulique souteraine ». La liste des rapports est d'ailleurs actuellement close et sera jointe aux bulletins d'inscription, qui seront diffusés en février 1960. Entre temps, la notice paille, que chaque membre a recue soit sous pli, soit encartée dans Mémoires et Travaux, donne un aperçu de ces rapports et quelques précisions sur le programme des voyages d'études associés aux Journées.

Nous prions nos collègues et amis intéressés par ce programme de se manifester sans tarder afin que le Secrétariat leur envoie les bulletins d'inscriptions.

\section{Publications}

Nous avons publié depuis la demière session le compte rendu des sessions de novembre 1958 et mars 1959 dans les numéros ci-après :

a) Session de novembre 1958: Quelques projets de grands aménagements hydrauliques dans le monde; La Houille Blanche, n* 4, 1959.
Nous rappelons que les communications présentés aux séances ordinaires de cette session ont été publiées comme suit :

- Dans le no II, 1958 de Mémoires et Travaux :

M. Gibrat : « Le Congrès atomique de Genève et l'Hydraulique $\gg ;$

et M. Ferrari : «Théorie du puits en écoulement non permanent $\gg$.

- Dans le $\mathrm{n}^{\circ}$ 1, 1959 de La Houille Blanche :

M. Schneebel. : « Sur l'utilisation de l'ail pour la mesure in situ de la perméabilité d'un terrain alluvial non saturé »,

ei dans le n* 3, 1959 de La Houille Blanche :

M. Egurazanofr : «Une solution du problème des débits solides, en tenant compte de l'influence de la couche de fond de grande concentration $»$.

b) Session de mars 1959 :

$1^{\circ}$ Travaux du Pool Coriolis : La Honille Blanche, $\mathrm{n}^{0} 5$, 1959 ;

2" Etudes des crues cévenoles (septembre-octobre 1958) : Mémoires et Travaux, no I, 1959.

Enfin, La Houile Blanche prépare la publication des mémoires ci-après :

- Dans le présent numéro (II, 1959) de Mémoires et Travaux, les mémoires présentés aux séances de juin 1959 par MM. Hermant et Wolf, Escande et Sananes, Lariev, Henry et Mauchamp, André, Poggr, et un l'ésumé de l'hydrologie de l'Hérault de Mlle Rougé (Prix Henri-Milon 1959).

Ce numéro sera complété par la publication :

$1^{\circ}$ Des mémoires de MM. Pardé eT Chantier, et do M. Robren, présentés en mars 1959;

2 Du présent comple rendu des travaux de la session des 19 et 20 novembre 1959 (Liste des participants, Vie de la Société, Documents regus).

(On trouvera les titres et la pagination de ces documents dans le sommaire du présent numéro.)

- Dans le $\mathrm{n}^{\circ}$ 6, 1959 de La Houille Blancine, les mémoires de M. Herman'T : « Application de la mesuro des débits par la méthode de dilution comparce à la détermination du rendement des turbines $\$$, présenté en novembre 1958

et de M. Chen Ghe Pen : « Lat différence de pertes de charge dans un écoulement en conduit circulaire, d'une part, et dans un ecoulement en canal i ciel ouvert à faible tirant d'eatu, d'autre part $»$, présenté en juin 1959.

-.- Dans le $\mathrm{n}^{\circ} 3,1960$, de La Houille Blanche, Io mémoire de MM. Coutagne et de Beavregard.

- Dans le $\mathrm{n}^{\circ}$ 2, 1960 de La Houille Blanche, les « Recherches récentes sur la houle », présentées en juin 1959.

(Pour la publication des mémoires de la session des 19 et 20 lovembre 1959 , voir a la fin du present compte rendu.)

\section{Congrès}

La VII Conférence on Coastal Engineering se tiendra du 22 au 27 août 1960 au Kurhaus à Scheveningen.

Les sujets traités au cours des séances de travail seront relatifs aux travaux techniques côtiels : formation et conduite de vagues et de houles, marées et ondes de tempête, formation et érosion des côtes, défense des cotes et construction de levées, aménagement et entretien de ports, etc., y compris les instruments et les méthodes utilisés pendant les recherches scientinques dans ce domaine. 
Des visites sont prévues aux travaux d'assèchement du Zuyderzéce el au Laboratoire d'Hydraulique de Delft.

Les frais d'inscription sont de fl. 20 et de fl. 10 pour les dames accompagnantes.

Les inscriptions seront prises soit :

- Chez le Professeur J. W. Johnson, University of California, Engineering Field Station, Building 159, Richmond 4, California,

- chez M. J.B. SchrJf, 25 Koningskade, La Haye (Hollande).

Les personnes désirant présenter une communication doivent le signaler a l'une ou l'autre des adresses précitées en précisant le títre du sujet traité et en y joignant un court résumé.

\section{Association Internationale}

\section{de Recherches Hydrauliques (A.I.R.H.)}

L'A.I.R.H. (Président : P. DANEL), dont plusieurs d'entre vous font partie, organise en France à Nice, en septembre prochain, un symposium sur la Recherche dans les turbines hydrauliques.

Ce colloque interfère avec nos activités, à un double titre: il se tient à une date voisine de nos Journées de Nancy et traite d'un sujet voisin de celui traité à Aix il $y$ a un an. Une coordination s'imposait. Elle est en cours. Le Comité local d'organisation de ce Symposium prévu par les statuts de l'A.I.R.H. sera le bureau de notre Comité Technique.

Les frais d'organisation n'incomberont en aucune manière à la S.H.F.

Après cet exposé de la «Vie de la Sociélé $»$, M. le Président Chapouther enchaîne avec la première partie de lordre du jour technique de la session :

\section{LA MÉCANIQUE DES FLUIDES ET LA MÉTÉOROLOGIE}

En rappelant que « la Météorologie » est un peu notre mère puisqu'il n'y aurait pas d'eau s'il n'y avajt pas d'atmosphère, M. Chapouthien passe la présidence à M. Joannès Thomas, Inspecteur général de la Météorologie, qui a bien voulu accepter de diriger les débats de cette séance.

La séance se poursuit sous la présidence de M. Joannès Thomas. Après avoir présenté les excuses de M. Viaut, Directeur de la Météorologie, que ses fonctions appellent en Asie pour quelques jours encore, M. le Président Joannès Thomas remercie la Société Hydrotechnique de France de linvitation qu'elle a faite à la Société Météo- rologique de France et de l'hospitalité qu'elle lui ofire en cette Journée du 19 novembre 1959 .

M. le président Joannes Thous se félicite du nombre, et si lon en juge par les titres, du haut intérêt des communications que tout le « brain-trust» de la Météorologic va présenter à ces séances.

Ensuite la parole est donnée, successivement, à MM. I ADY et Rovir.eaU qui présentent les communications ci-après :

\section{Caractères particuliers de la Mécanique des fluides en Météorologie,}

par M. DADY, Ingénieur de la Météorologie, Etablissement d'Etudes et de Recherches Météorologiques.

Avant de donner la parole à M. DADY, M. le Président l'a présenté à l'auditoire en ces termes:

«C'est actuellement le météorologiste le plus envié de tous ses collègues. En effet, si l'on devait jouer le jeu des définitions, je dirais que M. DADY est actucllement « un cerveau dans un cerveau $»$, parce que nous attendons, cette année, à la Météorologie, l'installation d'une machine èlectronique que nous affecterons à la prévision du temps; or, cette machine électronique, si ses locaux sont prêts, n'est pas encore dans les locaux : elle est dans le cerveau de M. DADY, d'ou la définition : « un cerveau dans un cerveau $\gg . »$

Les grands problèmes de la Météorologie : difficultés physiques et difficultés théoriques,

par M. J. Roulleau, Inspecteur général de la Météorologie, Chef de l'Etablissement d'Etudes et de Recherches Météorologiques.

Avant de donner la parole à M. Rounuad, M. le Président l'a prẻsenté en ces termes :

«... il est chargé, à la Météorologie Nationale, à la fois de l'ensemble des études et des recherches scientifiques et de l'Ecole de la Météorologie : autrement dit, il est responsable pour plusieurs années de notre ravitaillement en idées et en hommes. C'est une lourde responsabilité, et quand l'on sait qu'il trouve encore le temps de se livrer à des recherches personnelles, c'est un sujet d'étonnement pour tous ceux qui le connaissent. 》

La séance se termine par la projection du film : «Air Masses and Fronts », réalisé par les Services de la Marine américaine et commenté par M. Mezis, Ingénieur en chef de la Météorologie, Directeur de l'Ecole de Météorologie.

La séance est levée à $11 \mathrm{~h} 45$.

\section{SÉANCE DU JEUDI APRĖS-MIDI 19 NOVEMBRE 1959}

La séance est ouverte a 14 h 30 sous la présidence de M. BARHILION.

Sont successivement présentées les communications ciaprès :

$1^{\circ}$ Formations nuageuses : étude d̀ grande échelle et aperçu sur la formation de gouttelettes,

par M. Bricard, Professeur à la Faculté des Sciences de Paris, Laboratoire de Physique des Nuages et d'Electricité atmosphérique.
M. le Président remercie M. Bricaro dont le sujet est très étendu et pourrait donner lieu à de vastes développements.

L'exposé de M. Brycard ayant un caractère didactique, M. le Président demande à M. Rounceat si cet exposé correspond bien à l'enseignement actuel de l'Ecole des Ingénieurs Météorologistes.

M. Roulleau répond que e'est tout à fait son opinion, puisqu'il envoie ses élèves chez M. Bricaro.

M. le Président indique alors que la S.H.F. considé- 
rema le très bon exposé didactique de M. Bricand comme « la loi et lés prophètes » dans ses relations avec la Métérologic. Il estime cependant qu'il serait utile de réunir une petite commission, qui comprendrait par exemple deux professeurs de météorologie et deux proiesseurs d'hydrologie pour s'entendre sur la terminologie et préciser quelques définitions de termes tels que stabilité, circulation, qui semblent être employés par les météorologistes dans un sens différent de celui que leur donnent les hydrauliciens.

\section{$2^{\circ}$ Circulation atmosphérique d̀ haute altitude,}

par M. Banbé, Ingénieur en chef de la Météorologic Nationale.

$3^{\circ}$ Perspectives de la prévision numérique des précipitations,

pax M. Pone, Ingénieur en chef de la Météorologie Nationale, Chargé de Ja Division «Plévision» au Service Météorologique de la Métropole et de l'Afrique du Nord.

\section{Diffusion par turbulence dans la couche limite du sol,}

par M. J. SAIsSAC, Ingénieur de la Météorologie, Etablissement d'Etudes et de Recherches Météorologiques.

\section{PUBLICATION DES MÉMOIRES (séances du 19 novembre)}

Les mémoires de MM. Dady, Barbé, Pone et Sarssac et le commentaire du film «Air Masses and Fronts 》 sur «La Mécanique des Fluides et la Météorologie » seront publiés dans le dernier numéro de la revue trimestrielle La Météorologie de l'année 1959. Un tirage à part de ce numéro sera adressé aux membres de la S.H.F.

La conférence de M. Bricard, qui consistait en un aperçu sur ses nombreux travaux déja publiés dans « La Météorologie » et dans la collection « Encyclopédie de la Pléiade $»$, n'a pas donné lieu à une nouvelle publication.

La séance est levée à 18 h 18 .

\section{SÉANCE DU VENDREDI MATIN 20 NOVEMBRE 1959}

La séance est ouverte à 9 h 15 sous la présidence de M. P. Chapouthier, Inspecteur général d'Electricité de France.

M. le Président prononce, les quejques paroles ci-après, en guise d'introduction :

«L'ordre du jour d'hier ètait consacré à la Météorologie.

* Dans un souci de variété, il a paru intéressant de consacrer la réunion de ce matin a des échanges de vues sur les Congrès qui ont eu lieu depuis notre dernière réunion.

« Nous avons eu notamment, ceux de Padoue, dont lun des thèmes fondamentaux était l'hydraulique fluviale et celui de l'A.I.R.H. tenu à Montréal.

"Plutot que de vous en faire un compte rendu pur et simple, dont l'aridité risquait d'être lassante, le Bureau de votre Comité Technique a demandé à trois congressistes d'appeler votre attention sur les themes principaux de leurs debats. Ils vont ainsi vous présenter quelques questions d'hydraulique telles qu'elles ont été mises au point dans ces réunions, leurs communications sont ainsi articulées:

- Pour Padone, sur l'hydraulique fluviale, qui sera présentée par M. ChaberT;

- Pour Montréal sur deux thèmes : un théorique et un pratique :

- la question des courants de densité sera présentée par M. VaLEMBOIS;

- M. Kamerlocher développera le point de vue de l'ingénieur d'Equipement, vannes et vibrations notamment.

« Notre ami, M. Tonin, a bien voulu déposer sur ce bureau quelques plaquettes rédigées en italien et en anglais : Notices descriptives du Centre de modèles hydrau- liques de Nove, rattaché à l'Université de Padoue. Elles sont à rotre disposition. $\gg$

« Je donne la parole ì M. Jacques CiHabenT. "

Les comptes rendus suivants sont successivement présentés :

- VIe Congrès d'Hydraulique et de Constructions hydrauliques. Padoue, 25 au 28 mai 1959 , par M. Jacques Chabert, Chef de Groupe au Laboratoire National d'Hydraulique à Chatou.

A l'issue de cette conférence, et un peu en annexe, M. Chanerer a projeté un petit film sur le chantier du barrage du Vaïont construit pour la Società Adriatica di Elettricitá.

- 8" Congrès de l'A.I.R.H., Montréal, septembre 1959: la question des courants de densité, par M. ValemBoIs, Chef de la Division Recherches au Laboratoire National d'Hydraulique à Chatou.

- $8^{\circ}$ Congrès de l'A.I.R.H., Montréal, septembre 1959 : problèmes d'équipement hydraulique et hydroélectrique, par M. Kammenlochen, Chef du Service électromécanique de la R.E.H. 8, E.D.F.

La séance est levée à 11 h 47 .

\section{PUBLICATION DES MÉMOIRES}

Les comptes rendus de Congrès présentés par MM. ChaBERT, Valembois et KaMmeriocher seront publiés dans le $n^{\circ}$ I, 1960 de Mémoires et Travaux et dans le $n^{\circ} \mathrm{A}$ de La Honille Blanche en 1960. 


\section{DOCUMENTS REÇUS DEPUIS LA DERNIÈRE SESSION}

\section{HYDROLOGIE}

GA 1. - B. BELl. - Main results of meteorological research done in Hungary during the years 1954-56. Separatum: Acta Technica Academiae Scientiarum Hungaricae, tome XVIII, fascicule 1-2, Budapest, 1957.

GA 1. - V. Frolow. - Prévision à très longue échéance. Changements climatiques résultant de l'érosion. Commission du Bassin de la Seine, U.G.Gr.I., Comité National Francais, Paris, 1959.

GA 1. - E. Sifontes. - Micro-clima de ciudad Bolivar. Hidrologia vemezolana. - Meteorologia tropical. Barcelone, 1958.

GA 1. - Anonyme. - Association Internationale do Météorologie et de Physique de l'Atmosphère. Statuts. U.G.G.I., Toronta, 1957.

GA 1. - Anonyme. - International Association of Meteorology and Atmospheric Physics. Report of proceedings.

International Union of Geodesy and Geophysics. XIth General Assembly, Toronto, September 1957. Publication IAMAP $\mathrm{n}^{\circ} 11 / \mathrm{b}$, Londres, mai 1958.

GA 11. - A. Carre. -- La formation du réseau hydrographique dans la zone bibanique et l'évolution glyptogénique du Tell méridional au nord du Hodna (Algerie).

Publications de la Carte géologique d'Algérie, bulletin $n^{\circ} 20$, Alger, 1958.

(Laboratoire de Géologie, Besançon.)

GA 11. - A. Came, - Les glissements sous-marins dans le sillon miocène sud-tellien et la mise en place de l'unité A.

Extrait des Publications de la Carte géologique d'Algérie, bulletin $\mathrm{n}^{\circ} 20$, Alger, 1958.

(Laboratoire de Géologie, Eesancon.)

GA 11. - A. Carre et P. Marie. - L'éocène dans le massif du Djebel Kasbah, coupe de Nador (Algérie). Pablications du Service de la Carte géologique de l'Algérie, Alger, 1957.

(Laboratoire de Géologie, Besançon.)

GA 11. - M. Dreyfuss. - Excursions géologiques dans le Jura. IV : Chaudanne, Rosemont et la Malcombe. Extrait de : Bulletin bimestriel de la Société d'Histoire Naturelle du Doubs, n*23, janvier-février 1959. (Laboratoire de Géologie, Besançon.)

GA 11. - M. Matrauer. - Sur l'âge tertiaire du poudingue de Montfaucon (Doubs).

Extrait du $C$. R. sommaire des séances de la Société Géologique de France, $1958, \mathrm{n}^{\circ} 2$. Séance du 20 janvier 1958.

(Laboratoire de Géologie, Besançon.)

GA 11, - N. Theobald. - Les poissons fossiles du permien inférieur de la. Sarre.

Extrait de : Annales Universitatis Saraviensis Naturwissenschaften Scientia, VII, 2, Saarbrücken, 1958. (Laboratoire de Géologie. Besançon.)

GA 11. - N. Theorald. - Contribution à l'étude des Stégocéphales du Permien inférieur de la Sarre.

Extrait de: Annales Universitatis Saraviensis Naturwissenschaften Scientia, VII, 2, Saarbrücken, 1958. (Laboratoire de Géologie, Besançon.)
GA 12 - N. Tschinhar't. - Les perturbations atmosphériques intéressant l'A.E.F. méridionale.

Monographies de la Météorologie Nationale, $\mathrm{n}^{\circ} 13$, Paris, juin 1959.

GA 123 - E. Sifontes. - El rocio tropical.

Higrologia venezolana. - Meteorologia tropical. Barcelone, 1958.

GA 132 - P. Monel. - Fusion de la neige. Centre Hudrométéorologique E.D.F., Lyon, 1958.

GA 132 - P. Moner. - Prévisions d'apports à la réserve de Roselend. Centre Hydrométéorologique E.D.F., Lyon, 1958.

GA 132 - A. Pogar. - La fusion de la neige et les crues de juin 1957 dans les Alpes françaises orientales. Extrait de la Revue de Géographie alpine, Grenoble.

GA 134 - Anonyme. - Etat des avalanches et de leurs dérgats pendant l'hiver 1958-1959 dans le département des Hautes-Alpes. Météorologie Forestière, Gap, 17 juillet 1959.

GA 1343 - J. Messines. - La montagne aussi est en péril.

Rivières et Forêts, $n^{\circ} 13,2^{\circ}$ trimestre 1959 , CrépinLeblond et Cie, Paris, 1959.

GA 1343 - P. Chinuss. - Travaux récents contre les avalanches dans les Fautes-Pyrénées.

Rivières et Forêts, no 13, Crépin-Leblond et Cie, Paris, 1959.

GA 1343 - L. ANCHienri. - Lutte contre les avalanches dans les Alpes de Savoie. Rivières et Forêts, no ${ }^{\circ} 13$, Crépin-Leblond et Cie, Paris, 1959.

GA 24 - NEpeco. - River studies and recommandations on improvment of Niger and Benue. North Holland Publishing Company, Amsterdam.

GA 25 - L. ANchierr. - Les inondations de juin 1957 dans les Alpes de Savoie, Maurienne et Tarentaise. Rivières et Forêts, ${ }^{\circ} 13$, Crépin-Leblond et Cie, Paris, 1959.

GA 25. - V. Frorow. - Signe de la diff'érence entre les cotes de deux crues consécutives à Paris (manuscrit : tableau). Envoi du 2 juillet 1959, Paris.

GA 25 - A. JaAnnin. - Les crues cévenoles de septembre-octobre 1958 dans le département du Gard. Rivières et Forets, ${ }^{\circ}$ 13, Crépin-Leblond et Cie, Paris, 1959.

GA 25 - M. PardÉ. - Sur les fontes des neiges lors des crues. La grande erue alpestre de juin 1957. Extrait de la Revue de Géographie Alpine, Grenoble.

GA 25 - A. Poggr. - La fusion de la neige et les crues de juin 1957 dans les Alpes françaises orientales. Extrait de la Revue de Geographie Alpine, Grenoble.

GA 25 - J. P. Rogí. - Les ravages des inondations de juin 1957 dans le Queyras (Hautes-Alpes).

Rivières et Forêts, $\mathrm{n}^{\circ} 13$, Crépin-Leblond et Cie Paris, 1959.

GA 4 - P. NYDEGGER. - Vergleichende limnologische Untersuchungen an sieben Schweizerseen. Beiträge zur Geologie der Schweiz.

Iydrologie, $\mathrm{n}^{\circ}$ ), Schweizerischen Naturforschenden Gesellschaft. Berne, 1957. 
GA 557 - J. Makowski, - The use of radioactive isotopes in hydroconstruction, hydraulics and soil nechanics.

Rozprawy Hydrolechniczne, Zeszyt 5, Polska Akadeinia Nauk, Varsovie, 1959.

Gid 63 -- S. Stennemann. - Experimentelle Untersuchungen zur Plastizität von Eis. - Beitrage zur Geologie des Schweiz.

Hydrologie, ${ }^{\circ} 10$, Schweizerischen Naturforschenden Gesellschaft. Berne, 1958.

GA 64 - O. Lanser. - Beitrïge zur Hydrologie des Gletschergewässer.

Schriftenreihe des Oesterreichischen Wasserwirlschaftverbandes, Heft 38, Springer-Verlag, Wien, 1959.

G.t 7 -G. Olschow et K. Kohler. - Naturnaher Ausbau von Wasserläufen Gleichartigen Arbeitstagung auf Bundesebene rom 10-12.10.1956 in Würzburg. Landwirtschaft Angewandte Wissenschaft.

V.D.G., n ${ }^{\circ}$ 79, Landwirtschaftsverlag G.m.b.H., Hiltrup bei Münster (Westf.).

GA 31 - E. Sifontes. - Manchas del Orinoco. - Las rocas pintadas.

Hidrologia venezolana, -- Mefeorologia tropical. Barcelone, 1958 .

\section{HYDRAULIOUE GÉNÉRALE}

GB 34 - K. HaindL. - Theory of the hydraulic jump in closed conduits and its use in pratic (résumé en anglais).

Vyzkumny Ustav Vodohospodarsky, sesit 98, Prague, 1958.

GB 36 - P. Basiaux, - Etude du dynamisme d'un jet d'eau torsadé.

19 octobre 1959 , Najac (Aveyron)

(iB 42 - J.-B. Merien. - Etude de l'écoulement des fluides dans les tubes circulaires. Manuscrit S.H.F., 1959.

GB 44 - K. HAINir. - Non permanent flow at the closure of a valve at the beginning of a pipe line (résumé en anglais). Vizkumny Ustav Vodohospodarsky, Prague, 1959.

GB 52 - J. Martinec. - The effect of river channel roughness on the velocity of flow (résumé en anglais). Vyzkumny Ustav Vodohospodarsky, sesit 96, Prague, 1958.

GB 61 - M. Carlier. - Application des méthodes graphiques à l'étude des régimes progressivement variables dans les réseaux de canalisations.

Ministère de l'Agriculture. Direction générale $d u$ Génie Rural et de l'Hydranlique agricole, Paris, 1959.

GB 63 - V. Frolow - Prévision à très longue échéance. Changements climatiques résultant de l'érosion. Commission du Bassin de la Seine. U.G.G.I., Comité National Français, Paris, 1959.

GB 932 - R. Pedroli. - Seilflugelanlagen fur die Vornahme von Wassermessungen. Extrait de Cours d'Eau et Energie, n 3 , 1949, Zurich.

\section{ÉTUDE TECHNOLOGIQUE}

DES PRINCIPAUX OUVRAGES UTILISES DANS LES AMENAGEMENTS HYDRAULIQUES

GC 103 - J. Fnitsch, F. Tremmer, A. Wognin. - Die Studienreisen. Der VI. Kongress der Internationalen Talsperrenkommission. Schriftenreihe des Oesterreichischen Wasserwirtschaftsverbandes, Heft 39, Springer-Verlag, Wien, 1959 .
GC 111 - E. Nonverler. - Stabilité des barrages non homogènes (r'èsumés en anglais, français et russe). Hidrotehnicki Institut « Ing. Jaroslav Cerni », Belgrad, 1957.

GC 119 - E. Tremmel. - Erhöhung von Sperren Spannungen und Formänderungen an Talsperren. Der VI. Kongress der Internationalen Talsperrenkommission. Schriftenreihe des Oesterreichischen Wasserwirtschaftverbandes, Heft 39, Springer-Verlag, Wien, 1959.

GC 122 - J. Firrsch. - Betontechnische Fragen. Der VI. Kongress der Internationalen Talsperrenkommission.

Schriftenreihe des Oesterreichischen Wasservirtschaftuerbandes, Heft 39 , Springer-Verlag, Wien, 1959 .

GC 133 - P. Basiaux-Defrance. - L'ogive et la parabole dans la construction.

Extrait de L'Onde, IX, 1958, Najac (Aveyron), 1958.

GC 133 - P. Basiaux-Defrance. - Barrage concave (résumé).

Najac (Aveyron), 1959

GC 135 - E. Nonverller. - Stabilité des barrages non homogènes (résumés en français, anglais, russe). Hidrotehnicki Institut « Ing. Jaroslav Cerni », Beograd, 1957.

GC 135 - A. Wogris. - Verdichtung von Dammbaumaterial. Der VI. Kongress der Internationalen Talsperrenkommission.

Schriftenreihe des Oesterreichischen Wasserwirtschaftverbandes, Heft 39 , Springer-Verlag, Wien, 1959 .

GC: 223 el GC $246-$ E. Rotten Kaprun. - Anwendung von Spritzbeton.

Schriftenreihe des Oesterreichischen Wasserwirtchaftsuerbandes, Heft 35, Springer-Verlag, Wien, 1958.

GC 233 -- K. Hainde. - Non-permanent flow at the closure of a valve at the beginning of a pipe-line (résumé en anglais). Vyzkumny Ustav Vodohospodarsky, Prague, 1959.

GC 242 - R. BEnsimon. - Les fontes (prospectus). Cours professé à l'Ecole Nationale Supérieule de l'Armement et à l'Ecole Nationale du Génie Rural. Eyrolles, Paris, 1959.

\section{HYDRAULIOUE \\ DES AMENAGEMENTS HYDROÉLECTRIQUES}

GD 0 - P. BasiauX-Defrance. - Quelques idées au sujet de l'équipement rationnel des chutes d'eau. L'Onde (Toulouse), 1942.

GD 01 - H. GRennga, - Entwicklung der Oesterreichischen Wasserkraftnutzung. (Evolution des besoins de l'Autriche en énergie hydraulique.)

Die Salzburger Tagung, 1959, Springer-Verlag, Wien, 1959.

GD 01 - H. LaUda. - Wasserwirtschaftliche Probleme der Industrie. (Les problèmes de l'énergie hydraulique dans l'industrie.)

Die Salzburger Tagung, 1959, Springer-Verlag, Wien, 1959.

GD 01 - G.A. Tondur. - Die Elektrizitätswirtschaft Skandinaviens: Vergleich mit den Verhältnissen in der Schweiz und ihren Nachbarländern. (L'ènergie électrique en Scandinavie, comparaison avec la situation en Suisse et dans les pays voisins.)

Tiré à part de Cours d'Eau et Energie, $n^{\circ} \overline{5}$, Zurich, 1959 . 
GD 1 - H. VoLKER, - Wechselbeziehungen zwischen Wasserwirtschaft und Schiffahrt. (Corrélation entre énergie hydraulique et navigation.)

Die Salzburger Tagung, 1959, Springer-Verlag, Wien, 1959 .

GD 12 - P. Basiaux. - Quelques notes sur la prospection hydraulique. L'Onde, Toulouse, 1925.

GD 13 - P. Basiaux. - Lacs pyrénéens et stations de pompage.

L'Onde, Centre d'études, Carcassonne, 1946.

GD 82 - Nebeco. - River studies and recommandations on improvment of Niger and Benue. North Holland Publishing Company, Amsterdam.

GD $82-$ F. Sowinski. - Small hydro-electric stations considered in relation to the resources of hydroenersy of rivers in Poland (résumé en anglais).

Rozprawy Hydrotechniczne, Zeszyt 5, Polska Akademia Nauk, Varsovie, 1959.

HYDRACLIQUE APPLIQUEE A LA CORRECTION DES RIVIERES
ET A T.A NAVIGATION

GE 118 - H. Vorker. - Wechselbeziehungen zwischen Wasserwirtschaft und Schiffahrt. (Corrélations entre énergie hydraulique et navigation.)

Die Salzburger Tagung, 1959. Springer-Verlag, Wien, 1959.

GE 24 - P. Bastaux-Defrance. - Polte d'écluse discoüdale (résumé).

Najac, 1959.

HYDRAULIOUE

APPLIQUEE AUX TRAVAUX MARITIMES

GF 01 - Anonyme. - Lucien Vantroys (1911-1959). E.D.F., Direction des Etudes et Recherches, Paris, 1959 .

\section{HYDRACLIQUE DES DISTRIBUTIONS D'EAU ET DES EGOUTS}

GH 0 - E. IKoneF. - Wasserwirtschaftliche Aufgaben der Gemeinden. (Le problème hydraulique des communautés (alimentation en eau potable).)

Die Salzburger Tagung, 1959, Springer-Verlag, Wien, 1959 .

GH 701 - G. Schwals, - Der Mensch und das Wasser. (L'homme et l'eau.)

Die Salzburger Tagang, 1959, Springer-Verlag, Wien, 1959.

GH 701 - Anonyme. - Problèmes européens de la protection des eaux.

Fédération européenne pour la protection des eanx. Publie avec l'aide du Gouvemement de la République Fédérale Allemande, Munich, 1957.

GH $701 \rightarrow$ Anonyme. $\rightarrow$ Technische Universität, Berlin : Verleihung der Ehrendoktorwürde der Technischen Universität Berlin an Herrn Direktor Dipl. Ing. Hans Konig, 9 février 1959.

\section{HYDRAULIQUE AGRICOIF}

GI 01 - E. Harmans. - Die Bedeutung des Wassers für die Landwirtschaft. (L'importance de l'eau pour l'agriculture.)

Die Salzburger Tagung, 1959, Springer-Verlag, Wien, 1959.

GE 126 - M. Carlier. - Application des méthodes graphiques à l'étude des régimes progressivement variables dans les réseaux de canalisations.

Ministère de l'Agriculture, Direction generale du Génie Raral et de l'Hydraulique agricole, Paris, 1959.

\section{DIVERS}

$\mathrm{X}$ - R. C. Brewer. - The numerical control of machine tools.

Engineers' Digest Survey, $1^{\circ} 5$, Londres.

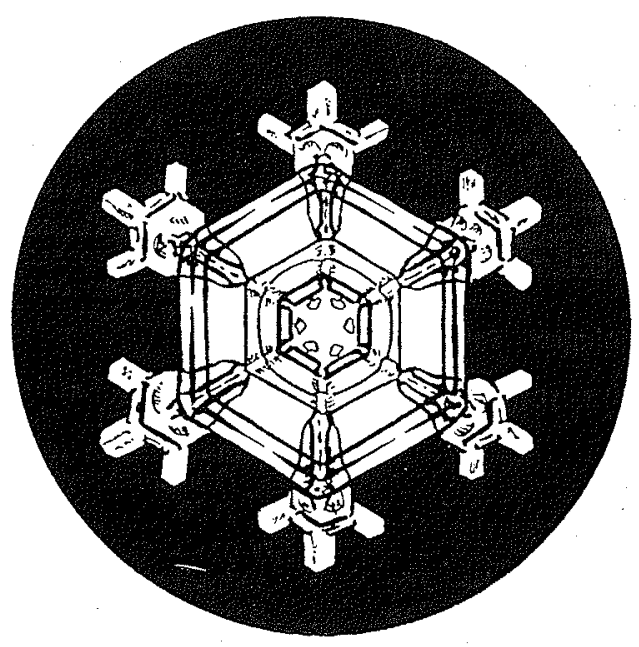

